\title{
LES ATTRIBUTS SYMBOLIQUES DE L'AUTORITÉ DU JUGE DANS LE DOCUMENTAIRE JUDICIAIRE
}

\begin{abstract}
Arnaud Lucien ${ }^{1}$
« La Cour. Ces mots nous firent lever d'un bond. Prononcés par l'huissier du palais de justice, ils annonçaient l'entrée des trois juges qui, têtes nues, en robes noires, pénétrèrent dans la salle par une porte latérale et prirent place sur la partie supérieure de l'estrade $[\ldots]$ juste en dessous des magistrats, se tenaient les traducteurs [...] Sur le gradin inférieur à celui des traducteurs se trouvait l'accusé dans sa cabine de verre. Il faisait face à la barre des témoins. De la salle on ne le voyait, de même que les témoins, que de profil. Au bas de l'estrade siégeaient enfin, le dos au public, le procureur [...] et la défense ».
\end{abstract}

Hannah Arendt, (1963), Eichmann à Jerusalem

L'autorité de l'institution judiciaire n'est pas sans lien avec la mise en scène qui l'accompagne. Même sécularisée, la Justice dans sa forme classique, conserve les ornements de pratiques ancrées dans le passé. Elle s'appuie sur un rituel fondé dans des mythes d'origines cosmogonique et religieuse qui nourrissent l'imaginaire de l'institution. Celui-ci trouve ses racines dans les grands procès de l'Histoire, de l'Antiquité et notamment d'épisodes bibliques tels que le jugement du Roi Salomon (Ost, 2004). Selon l'analyse de l'illustre anthropologue René Girard

1 Arnaud Lucien est Docteur en sciences de l'information et de la communicatio au Laboratoire «Information, Milieux, Médias, Médiations » EA 3820, Université du Sud Toulon Var.

Recherches en communication, $\mathrm{n}^{\circ} 28$ (2007). 
(1972), l'audience s'affirmerait comme la reproduction d'un sacrifice ; La sentence portant la signification de la vengeance collective. Par la répétition d'un rituel ancestral, le palais de Justice est donc un temple et l'audience une cérémonie. Cette présence du sacré dans les pratiques actuelles dispose d'un pouvoir performatif. En ce sens, le procès est une scène sur laquelle les interactions obéissent à des règles précises tant dans le respect des garanties procédurales que dans la répétition d'un rituel quasi-religieux. Le rituel, respecté, s'intègre dès lors dans un système symbolique à l'origine d'un invisible structurant et, derrière la mise en scène esthétique de la Justice, ce sont les symboles qui communiquent. Revêtu de sa robe, le juge incarne une fonction symbolique qui selon une approche psychanalytique se réalise dans l'exercice de la fonction paternelle. Il énonce l'interdit. C'est-à-dire à la fois la prohibition, la prescription et un dire d'interposition : une médiation entre la parole de la société, la parole divine et les Hommes. La dramatisation théâtrale, l'apparat de la salle d'audience, les costumes, l'organisation de l'espace et du temps participent d'un discours presque ésotérique. Dès lors, il est aisé de comprendre la pudeur de l'institution face à l'intrusion des caméras. En effet, en France, depuis la loi du 6 décembre 1954, l'enregistrement et la captation des débats judiciaires, sont par principe prohibés pour préserver la sérénité de l'audience. Une exception dite « historique » afin de constituer des archives était accordée par la loi dite Badinter du 11 juillet 1985. Elle permettait de justifier l'enregistrement des Procès pour crime contre l'humanité de Maurice Papon (1998), Klaus Barbie (1987) et Paul Touvier (1994). Par ailleurs, des autorisations sont exceptionnellement accordées avec le consentement des parties par le premier président de la Cour d'Appel du ressort de la juridiction concernée. Ce qui était le cas en juin 2009 dans l'affaire de l'explosion de l'usine AZF à Toulouse. En 2001, Raymond Depardon bénéficiait du sésame pour réaliser son chef-d'oeuvre : $10^{\circ}$ chambre instants d'audience. Il filmait l'activité d'un tribunal correctionnel dans le but de présenter un témoignage fidèle de l'audience. Plus tard, les réalisateurs de la série de documentaires « Nos juges » (Teianu I. et al., Lebas S. , Puech R. ; 2007) profitaient de l'autorisation pour saisir l'intimité de jeunes magistrats, leur humanité, leurs forces et leurs faiblesses. L'intérêt suscité par le monde judiciaire est progressivement devenu une véritable pression pour l'entrée des caméras dans les prétoires. En ce sens, le legal genre occuperait jusqu'à un tiers du temps de programmation des chaînes de télévision américaines (Perreur, 2003, p.56). Face à l'insistance des médias et de leur public, en 2004, un 
groupe de réflexion composé de personnalités du monde de la Justice et du monde des médias était créé par le Garde des sceaux sur la question de la captation et la diffusion des débats judiciaires. De nombreuses questions étaient soulevées, notamment celle de la fidélité des enregistrements d'audience, celle des droits de la personnalité des parties, de la sérénité des débats et d'une manière sous-jacente, la crainte d'une atteinte à la figure du juge était perceptible. En effet, la représentation médiatique emporte avec elle le risque d'atteinte à l'intégrité de la mise en scène qui accompagne l'acte juridictionnel. La première page du rapport Linden fait explicitement référence à ce danger en évoquant Marshall Mc Luhan, « [...] le medium est le message [...] la télévision crée son propre univers, fonde et affirme sa propre légitimité [...] elle produit sa propre vision du monde prise en compte par tous ». Il est dès lors justifié de s'interroger sur les effets de la médiatisation de l'institution judiciaire ? La captation des débats comme toute représentation fictionnelle s'expose à l'incomplétude commune à la représentation et s'accompagne nécessairement d'une narration parfois concurrente à celle du dispositif judiciaire [Partie $1:$ La concurrence des récits] en ce qu'elle fragilise ou disqualifie la fonction symbolique du juge [Partie 2 : Le juge réincarné].

\section{La concurrence des récits}

L'institution judiciaire, véritable théâtre des passions humaines, constitue une scène privilégiée pour l'œil de la caméra, cependant, la narration du documentaire a vocation à entrer en concurrence avec ce récit. Le documentaire le plus objectif dispose toujours d'un caractère fictionnel. En effet, pour des questions de format et d'intérêt, il est impossible de tout montrer. Il faut choisir les scènes les plus pertinentes pour atteindre la durée recherchée et sélectionner les passages susceptibles de retenir l'attention du public. Les choix techniques influencent le discours. Le réalisateur est confronté au paradoxe de devoir appréhender l'espace filmique tout en restant discret dans la salle d'audience. Raymond Depardon (2001) multiplie les stratagèmes pour se faire oublier. Dans $10^{\circ}$ chambre, instants d'audience, il habille le pied de la caméra, se revêt de noir et tente de camoufler les perches destinées à prendre le son. En ce sens, le rapport Linden ( p.8) envisage l'intrusion de la caméra comme l'immixtion d'un tiers, susceptible de modifier les comportements des acteurs de l'audience : «[...] il a été rappelé que la présence d'un tiers aussi prégnant qu'une caméra de télévision 
peut modifier le comportement des uns et des autres lors du procès, en provoquant soit des dérives de starisation qui peuvent atteindre tout aussi bien les magistrats que les avocats ou leurs clients, soit des comportements excessifs qui auraient pour finalité d'instrumentaliser le procès au bénéfice de certains acteurs de celui-ci ». En effet, la caméra instaure une autre scène, susceptible d'influencer la narration et les jeux d'acteurs. Elle se substitue à toutes les fonctions du cadre symbolique comme celle d'attribuer à chacun sa place. Le changement de comportement est perceptible dans le documentaire Délits flagrants de Raymond Depardon (1994). Au cours d'une scène présentant le déferrement d'une personne pour des faits de vol de véhicule, à la vue de la caméra, la personne mise en cause interroge le Procureur, «ça va je suis bien coiffée ? », la réponse du substitut organise un retour à l'ordre symbolique : «Vous savez c'est un film documentaire, ce n'est pas destiné à vous mettre en valeur vous [...] ». La caméra peut ainsi porter atteinte à la sérénité des débats à la fois dans la mesure où la médiation du discours de l'institution judiciaire entre en concurrence avec la médiatisation de la caméra mais aussi car elle modifie les comportements des acteurs de l'audience. On peut dès lors imaginer un magistrat plus sévère ou plus complaisant face à la caméra.

Du cadrage au montage, le réalisateur est l'auteur d'un récit, une mise en langage des images et des sons. Son point de vue est nécessairement exprimé : il s'agit du «projet du documentaire », ce qui est appelé scénario pour une œuvre fictionnelle. Un nouveau genre met en avant ce caractère narratif à travers le docufiction. Il s'agit alors de la forme romancée d'une affaire judiciaire. Une mise en scène qui, par ailleurs suscite les contentieux, c'est notamment le cas pour l'affaire Grégory Villemin (2006) relatant le meurtre d'un enfant et l'affaire Yann Piat (2009) à propos de l'assassinat d'un député. En ce sens, la même audience s'expose à une infinité de points de vue. De la réalité sociale du magistrat à l'esthétique du rituel judiciaire, le procès s'offre à la multitude des regards. Par ailleurs, la vérité judiciaire n'est pas nécessairement la vérité recherchée par le documentaire. Proposer un documentaire ne revient pas à refaire le procès, tout l'intérêt réside justement dans le regard exprimé et la sélection des scènes. Le rapport Linden (pp.5-6) évoque ce caractère narratif dans lequel le montage prend une place particulièrement apparente : «Le récit judiciaire privilégie naturellement la recherche loyale de la vérité ; $[. .$.$] Le récit$ médiatique est plutôt tourné vers les scènes marquantes de l'audience 
ou celles qui lui paraissent telles, même si elles ne sont pas les plus importantes pour la décision $»$. Certains documentaires comme celui de Raymond Depardon (2005), $10^{\circ}$ chambre Instants d'audiences, insistent particulièrement sur les attributs symboliques de l'autorité du Juge. D'autres réalisations s'attachent au contraire à réincarner le juge. C'est-à-dire le situer en dehors de sa fonction symbolique. Il s'agit de saisir la vérité sociale du magistrat. L'activité juridictionnelle est alors replacée dans un contexte plus intime, parfois même, tenant à la vie familiale du Juge. Dans $10^{\circ}$ chambre, au contraire, Raymond Depardon limite son champ au territoire de la salle d'audience pour saisir le juge dans sa fonction. Il use de plongée et de contre-plongée pour insister sur la dialectique opposant l'autorité de la Présidente de Chambre filmée en contre-plongée et la situation hasardeuse du mis en cause filmé en plongée. Par ailleurs, il arrive souvent que le magistrat n'apparaisse pas dans le champ de la caméra et que seule sa voix soit perceptible, lui conférant une certaine forme d'irrationalité propre à la mise en scène classique de l'acte juridictionnel. Cet aspect était clairement relevé par la Présidente de la $10^{\mathrm{e}}$ chambre. Selon ses propos : «Raymond a fait ce qu'il fallait pour qu'on la voit bien l'autorité [...] j'ai découvert des stratagèmes en voyant le film pour la troisième fois, cela consiste à ne pas me montrer quand je pose la question [...] elle n'arrête pas, elle interrompt, elle interroge, à l'impératif [...] on ne la voit pas [...] c'est une mitrailleuse ». Les deux manières de présenter le juge sont antagonistes, la première en dévoilant l'intimité du Juge, l'affaiblit dans sa fonction symbolique alors que la seconde insiste sur l'imaginaire de la figure du Juge et donc sur son autorité. Le déroulement de la décision judiciaire ellemême s'inscrit dans une narration comparable à celle de l'œuvre de fiction. Roland Barthes distingue deux modèles qui sont applicables à la narration judiciaire : la phrase herméneutique et l'intrigue de prédestination. Le premier repose sur la construction de l'intrigue, le spectateur découvre progressivement les différents éléments de la fiction jusqu'au dénouement. Dans le second modèle, les faits sont établis dès le départ, et la narration consiste à expliquer les faits dévoilés initialement. Ces modèles de narration sont comparables aux systèmes judiciaires. Le système accusatoire, adopté par les pays de common Law se rapproche de ce que Barthes appelle la phrase herméneutique, les parties sont maîtres du procès, elles convoquent les témoins à charge et à décharge et s'affrontent à l'audience, le juge ne disposant que d'une position d'arbitre. Ce sont les parties qui par leurs débats vont fournir au juge les pièces d'un puzzle qu'il devra 
ensuite organiser. Dans le système inquisitoire, ou modèle continental, le rôle des parties est moindre, le juge qui dispose de larges pouvoirs d'enquête a la charge de confirmer l'évidence de faits établis, ce qui correspond à une forme d'intrigue de prédestination. Il s'agit d'expliquer ce qui a conduit l'auteur à commettre le crime.

Ainsi, la représentation médiatique ne peut s'émanciper d'une mise en scène, d'une narration et dans le récit, la fonction symbolique du juge est appréhendée d'une manière contingente. Si le documentaire offre régulièrement au public un regard sur ce que veut être l'institution judiciaire dans sa forme classique, certains documents adoptent une approche différente et même concurrente au récit judiciaire, par la réincarnation du juge. C'est-à-dire en le dépouillant de sa fonction symbolique pour le saisir dans sa réalité sociale.

\section{Le Juge réincarné}

Le documentaire s'inscrit dans une finalité démocratique liée à la connaissance par les citoyens du fonctionnement de l'état. Par ailleurs, le rapport Linden (p.6) le rappelle, "La Justice dans une démocratie est nécessairement publique. [...] (elle) doit accepter d'être évaluée et de s'exposer au regard public ». L'entrée des caméras dans les prétoires ne peut être envisagée sous ces seuls aspects, l'immédiateté de l'image et l'angle adopté, peuvent représenter un danger pour l'autorité des institutions. La caméra est susceptible de porter atteinte à l'intégrité de la justice dès lors qu'elle affaiblit la fonction symbolique de ses représentants. Ainsi, dépouillé de sa robe et de l'apparat de l'audience, le magistrat est sorti de son rôle. Il y a crise de la représentation. Selon Daniel Bougnoux, celle-ci est manifeste quand les acteurs ne jouent plus un rôle, « quand la chose revient à la place du signe pour déloger celui-ci, pour le bousculer » $(2006$, p.8). En effet, là où la scène judiciaire organise une mise à distance, une coupure sémiotique, entretient une differ(a)nce par un langage, par un dispositif, par les signes, par le symbole, les médias cassent cette frontière confortable pour faire réapparaître l'homme en dehors de sa fonction symbolique. C'est-à-dire en le privant de son rôle social. L'institutionnalité est mise de côté pour saisir les coulisses d'une mise en scène destinée à porter des valeurs. Autorité, infaillibilité, rigueur, sagesse, force etc. laissent place à l'humanité dans ses faiblesses : Orgueil, incertitude, erreur, émotion etc. La dialectique puissance de la fonction / faiblesse de l'homme apparaît 
alors clairement. Ainsi, dans de nombreux documentaires tels que ceux de la série « Nos juges » ( Teianu, 2007 ; Puech, 2007 et Marant, 2007), et d'une manière générale dans les médias, le magistrat est personnifié, nommé par son nom. Son parcours, sa vie privée, ses traits de caractères sont exposés. C'est l'individu, homme ou femme, qui retient l'attention de la caméra. L'objet est probablement de rapprocher le magistrat du citoyen. L'institution judiciaire dans sa rigueur semble froide et éloignée des justiciables ; Présenter le juge d'une manière plus familière permettrait dès lors d'améliorer les rapports entretenus avec une institution pudique et inconnue des profanes. Le but est-il atteint ?. La proximité recherchée mérite souvent d'être requalifiée en familiarité. Le juge réincarné, personnifié et starifié, joue un autre rôle que celui qui est exigé de sa fonction. L'objet est de faire entrer le spectateur dans l'intimité du personnage. Le juge est présenté dans son quotidien et sa vie de famille. La faiblesse de l'homme, et désormais souvent de la femme, est exposée : les incertitudes ressenties face à la complexité d'un dossier sont accentuées et mises dans la perspective de la fragilité de la décision humaine. Le réel recherché n'est pas celui de la fonction mais celui d'une vérité sociale. La critique est d'autant mieux exprimée qu'elle est soutenue par la promesse de la vérité de l'image. À travers la médiatisation et la personnification du juge, l'objectif du metteur en scène semble être de provoquer une empathie, d'entrainer une identification ou un rejet. La frontière symbolique s'effondre. Le juge devient un homme ou une femme comme les autres, et finalement, c'est la fonction qui est affaiblie alors que le projet est de connaître le magistrat afin qu'il soit mieux compris, mieux respecté. L'ob-scénité de la représentation médiatique fait ainsi disparaître la nécessaire distance symbolique qui doit régir la pragmatique existant entre le citoyen et son juge. Ce dernier représente une institution qui doit être honorée et respectée comme telle. Selon la fable de La Fontaine, «L'âne qui portait des reliques » [titre initial de l'ouvrage d'Antoine Garapon intitulé Bien juger], la personnalité et l'individualité du juge, importent peu dans le cadre de la relation qui doit exister entre le juge et la société. C'est la fonction qui doit être respectée pour ce qu'elle est, dans le respect de la convention originelle. La personnification s'accompagne alors généralement d'un effet star et plus tard de l'affaiblissement de la fonction liée à la réincarnation. Ainsi, la chronique judiciaire rappelle toujours le nom et le prénom du magistrat et parfois ses précédents faits d'armes. Dès lors, ce n'est plus l'institution judiciaire qui intervient dans l'espace médiatique mais les juges Eva Joly, Laurence Vichnievski, Renaud Van Ruymbeke, Jean Louis 
Bruguière, Philippe Courroye, éric Halphen, éric de Montgolfier etc. Ils sont ensuite affublés de qualificatifs : Juge anticorruption, petit juge, juge rouge, croisé, etc. , font l'objet d'ouvrages, de films, et parfois, les écrivent euxmêmes. Ils participent à une vie publique, en tant qu'auteur, ou encore en tentant une carrière politique. Mais surtout, ils prennent une distance avec l'institution ellemême, en exprimant les divergences qui les opposent à leur hiérarchie. Le rapport entre le juge et la caméra est alors un rapport ambivalent. La satisfaction de l'ego des premiers temps cède souvent la place à la pression de l'opinion publique et au sentiment d'être jugé. La réincarnation du magistrat dans les médias en fait une cible individuelle qui n'est plus protégée par sa fonction symbolique. Ce n'est plus le juge d'instruction qui prend les décisions, mais l'homme : L'exemple est significatif pour l'affaire d'Outreau. En niant l'institutionnalité représentée par le juge, pour le saisir en tant qu'homme, c'est alors l'ordre symbolique qui est atteint.

Paradoxalement on s'aperçoit que c'est dans la fiction que la fonction symbolique du juge est la mieux mise en valeur, la romance reposerait même sur l'idéalisation du rôle représenté par les personnages du récit. Ces derniers incarnent en effet une fonction mise à l'épreuve de considérations axiologiques qui rappellent la norme, la différence entre le bien et le mal, et finalement présentent, parfois a contrario, l'idéal d'une fonction symbolique. Les exemples de la mise en scène des attributs symboliques du juge sont nombreux dans le cinéma, qui utilise le cadre judiciaire comme espace de l'intrigue. Nicolas Schmidt le souligne (2002, p.75), à l'écran, « les gens de robe dominent de leur autorité d'institution, leur communauté et leur famille, surtout tant qu'ils peuvent décider de la tête d'un homme ». Cette représentation d'une autorité institutionnelle ne souffre pas les frontières. En effet, l'imaginaire de l'institution judiciaire est fortement influencé par les représentations anglo-saxonnes qui éduquent à leur manière les téléspectateurs. Sabine Chalvon-Demersay (95) constatait déjà cet effet dans le domaine médical à travers la série télévisée Urgence. Un grand nombre de séries américaines sont diffusées en France. Il en est ainsi de Perry Mason, d'Ally Mc Beal, Les associés, La loi de Los Angeles, Matlock, Law and Order, Murder One, Jag, The Practice etc. de même, le cinéma américain dispose d'une grande popularité en France notamment avec des œuvres telles que Douze hommes en colère, Larry Flint, L'associé du diable etc. Les confusions fréquentes commises à l'audience traduisent le succès du genre. Il n'est pas rare, en effet, au 
cours du procès, en France d'entendre les parties s'adresser au magistrat en l'appelant « votre honneur » ou en s'exclamant « objection ». Alors que dans le procès continental le magistrat doit être appelé «Monsieur le Président » et que le système inquisitoire interdit toute intervention pendant les plaidoiries de la partie adverse. L'autorité institutionnelle du juge repose alors sur son pouvoir mais aussi sur l'imaginaire qui l'entoure. Barbara Villez (2007) dans son étude sur les séries télévisées consacrées à la Justice souligne la mise en évidence des attributs de l'autorité du juge, présents jusque dans son cabinet, par « des reliures rouges à lettres « (pp. 131 et 134). Cas assez marginal, elle relève le risque de sortir le juge de sa fonction pour lui donner celle de victime, en citant un épisode de la série télévisée The practice : «Il est étonnant de voir celle-ci [le juge] révéler à toute la Cour qu'elle a subi le même préjudice que dans l'affaire traitée, alors que cette confession la place en situation délicate, voire la fragilise et remet en question son impartialité ». En toute hypothèse dans la fiction, les attributs symboliques de la figure du juge semblent mieux préservés car le caractère spectaculaire de la Justice et son apparat, bénéficient naturellement à l'intrigue.

\section{Conclusion}

L'autorité de l'institution judiciaire repose sur une mise en scène classique qui accompagne l'acte juridictionnel et qui porte des valeurs : Force, impartialité, indépendance etc. Le récit judiciaire ne peut dès lors être séparé de son contexte d'énonciation. En ce sens, la narration organisée par la représentation médiatique a vocation à entrer en concurrence avec le discours institutionnel. Le documentaire s'affirme ainsi souvent comme un danger. La fonction symbolique du juge y est représentée de façon contingente. Si certains documents placent l'emphase sur les attributs symboliques de la fonction comme le cérémonial et l'apparat du palais, d'autres cherchent à saisir la réalité de l'homme ou de la femme, incarnée sous la robe, c'est-à-dire à briser la distance séparant le juge du justiciable. La réincarnation du magistrat expose dès lors ses faiblesses et fragilise l'institution. La représentation médiatique de l'acte juridictionnel implique donc nécessairement une démarche éthique qui prenne en considération l'importance de la fonction symbolique du juge pour le lien social. Selon Pierre Legendre, (2000, p.9-10) « Il est question du radeau institutionnel qui permet à l'humain, dans toute culture, de traverser la vie ». 


\section{Bibliographie}

Aumont, J., et Marie M., (2006). L'analyse des films, Paris : Armand Colin.

Baudry, JL., (1975). Le dispositif : approches métapsychologiques de l'impression de réalité, Communications, 23, 56-72.

Barthes, R., (1964). Essais critiques, Paris : Le seuil.

Bougnoux, D., (2006). La crise de la représentation Paris : La découverte.

Boutaud, JJ., (1997). Sémiotique et communication, du signe au sens, Paris : L'Harmattan.

Commission parlementaire (2005). Rapport de la commission sur l'enregistrement et la diffusion des débats judiciaires, 22 février 2005, dit rapport Linden, http:// lesrapports.ladocumentationfrancaise.fr/BRP/054000143/0000.pdf,

Chalvon-Demersay, S., (95). La confusion des conditions. Une enquête sur la série télévisée Urgences », Réseaux, 95, 235-283.

Derrida, J., (1979). L'écriture et la différence, Paris : Éd. Le Seuil.

Foucault, M., (1975). Surveiller et punir, Paris : Gallimard.

Garapon, A., (1996). Eschyle, Kafka, O.J. Simpson : Genèse et corruption du rituel judiciaire, Dans G. Pineau, (Éd) 2003, La justice saisie par la télévision, Dossiers de l'audiovisuel, 107, ( pp.6-8). Paris : La Documentation Française.

Garapon, A., (1997). Bien juger, Paris : Odile Jacob.

Gauthier, G., (2005). Le documentaire, un autre cinéma, Paris : Armand Colin.

Goffman, E., (1973). La mise en scène de la vie quotidienne, la présentation de soi, Paris : Éd de Minuit.

Lamizet, B., (1997). La médiation politique, Paris : L'Harmattan.

Lamizet, B., (2006). Sémiotique de l'évènement, Paris : Hermès Lavoisier.

Leblanc, G., (1995). Du modèle judiciaire au modèle médiatique, Dans G. Gauthier, A. Gosselin, J. Mouchon, Communication et politique Hermès, 17-18, (pp.63-72), Paris, CNRS éditions.

Legendre, P., (1999). Sur la question dogmatique en occident, Paris : Fayard.

Legendre, P., (2000). Le crime du caporal Lortie, Paris : Flammarion.

Lucien, A., (2007). Médiation et modernité, approche communicationnelle de l'institution judiciaire, Thèse en sciences de l'information et de la communication. Disponible à http://i3m.univ-tln.fr/-Theses-soutenues-.html

Lucien, A., (2008). La Justice mise en scène, Paris : L'Harmattan.

Lyotard, J-F., (1977), The unconscious as Mise en scène, Dans M. Benamou et C. Caramella (Éd.), Performance in postmodern culture, (pp. 86-98), Winsconscin : Coda Press.

Metz, C., (1997), Le signifiant imaginaire, Paris : C. Bourgois.

Ost, F., (2004). Raconter la Loi, aux sources de l'imaginaire juridique, Paris : Odile Jacob.

Perreur , N., (2003). Crime et Justice à la télévision américaine : ambivalence des représentations dans les tabloïds et les néo-séries, Dans G. Pineau, (Éd) 2003, La justice saisie par la télévision, Dossiers de l'audiovisuel, 107, ( pp.56-58). Paris : La Documentation Française.

Schmidt, N. (2002). Le monde de la Justice dans le cinéma français, Cinémaction, 105, 7486.

ZiZek, S., (2004), La subjectivité à venir, essais critiques, Paris : Flammarion. 


\section{Documentaires}

Depardon, R., (1994). Délits flagrants ; Arte vidéo ; Durée 1h 49.

Depardon, R.,(2005). $10^{\circ}$ chambre, instants d'audiences. Arte vidéo, Durée $1 \mathrm{~h} 45$

Marant, A., (2007). Juge d'Instance : Le Tribunal de la vie ordinaire, Capa, 53 min.

Puech, R., (2007). Juge d'Application des Peines : La liberté sous conditions, Capa 53 min.

Teianu, I., Lebas, S., Puech, R., (2007). Juge aux Affaires Familiales : le Tribunal du désamour, Capa, $53 \mathrm{~min}$. 
